\title{
Evaluation of Colorectal Surgical Patients and Treatment Modalities in a COVID-19 Pandemic Hospital: A Cross-sectional Study
}

\author{
Muzaffer Akinci ${ }^{1}$, Hilmi Bozkurt ${ }^{2}$, , Hüda Ümit Gür ${ }^{1}$, Adil Koyuncu ${ }^{1}$, \\ Mahmut Said Değerlii ${ }^{1}$, Sercan Yüksel ${ }^{1}$, Orçun Alpay ${ }^{1}$, Ahmet Kocakuşak ${ }^{1}$
}

${ }^{1}$ Department of General Surgery, University of Health Sciences, Haseki Resarch and Education Hospital, Istanbul, Turkey

${ }^{2}$ Department of Gastrointestinal Surgery, University of Health Sciences, Haseki Resarch and Education Hospital, Istanbul, Turkey

\section{*Correspondence}

hilmibozkurt27@gmail.com

(Hilmi Bozkurt)

\begin{abstract}
Objectives: The COVID-19 pandemic has emerged as a significant threat to healthcare systems, and has resulted in treatment algorithms being changed in general surgery clinics, as is the case in all other medical disciplines. In the present study we analyze the outcomes of patients who have undergone elective colorectal surgery in our hospital since the COVID-19 outbreak, as well as the precautionary procedural changes that have been made. Methods: The present study evaluates the approaches applied for the treatment of elective colorectal cancer patients in a pandemic hospital. In this cross-sectional study, conducted in a tertiary-level public hospital in Istanbul, Turkey, a retrospective review is made of the files of patients who underwent elective colorectal surgeries between 11.03.2020, as the date on which first case of COVID-19 was officially identified in Turkey, and 01.05 .2020 , in the general surgery clinic of a tertiary healthcare facility that has been designated a pandemic hospital. Results: A total of 18 patients underwent a surgical resection in accordance with oncological principles between the defined dates, having been diagnosis with a colorectal malignancy. None of the operated patients were considered suspicious for COVID-19 preoperatively, nor did they have any contact history. Furthermore, there were no suspicions or signs of COVID-19 during the 14-day follow-up of the discharged patients. Conclusion: It was established in the present study that patients undergoing colorectal surgery and patients with COVID-19 infections in the same hospital can be treated successfully without cross-infection through the application of appropriate isolation principles. These successful findings were supported by a 14-day follow-up after discharge.
\end{abstract}

\section{Keywords}

COVID-19, Colorectal surgery, Pandemic hospital

\section{Introduction}

COVID-19 (new type of coronavirus, 2019-nCoV), a viral disease that emerged in China in December 2019 and that is now known to spread through close contact, has since been declared a pandemic [1]. On January 7, 2020, a novel coronavirus $(2019-\mathrm{nCoV})$ was identified that had not been previously identified in humans, and the $2019-\mathrm{nCoV}$ disease was subsequently named COVID-19[2]. As COVID-19 spreads around the world and throughout our country, the hospitals in which the disease is being treated have become high risk areas for infection $[3,4]$. The first case was identified in our country in March, and by May 1, 2020, the number of cases in the country had reached 122,392 , approximately $60 \%$ of which were in Istanbul, where our hospital is located. Our hospital was one of the first four hospitals to be declared a pandemic hospital in the city, having been selected due to the high prevalence of COVID-19 patients in the vicinity [5]. With the increasing incidence of the disease in our country, a rapid increase has been witnessed in the number of confirmed or suspected COVID-19 patients in our inpatient wards. Within just a few weeks, more than $80 \%$ of our 700 -patient capacity hospital had been filled, and patients had been diagnosed with COVID-19. The postponement of elective surgery is recommended when possible in the regions hit worst by the pandemic [1, 4]; while for surgeries that are urgent and that cannot be delayed, immediate surgery is recommended after the necessary precautionary arrangements have been made $[6$, 7].That said, the method and timing of operations for colorectal cancers are still a question of debate in pandemic regions [8]. Owing to the speed at which the disease spreads and its effect on healthcare systems worldwide, hospitals are having to provide treatment to both COVID-19 patients and cancer patients for whom postponement may be problematic simultaneously. Upon the declaration of our facility as a pandemic hospital, a large proportion of the inpatient bed capacity was allocated to 
COVID-19 patients, while the operations required by cancer patients continued to be carried out in a controlled manner.

The present study evaluates the records of patients who have undergone elective colorectal surgery in our hospital since the COVID-19 outbreak, as well as the precautionary procedural changes made.

\section{Methods}

A cross-sectional evaluation is made of the elective colorectal cancer surgeries carried out in the general surgery clinic of a tertiary hospital between 11.03.2020, when the first official case of COVID-19 in Turkey was reported, and 01.05.2020. Cases involving emergency surgeries and non-colorectal malignancy surgeries were excluded from the study. The evaluated parameters included demographic characteristics, comorbidities, indications for surgery, preoperative patient assessment environment, preoperative imaging methods, infection parameters, surgical procedures, anaesthetic procedures, postoperative intensive care requirement, length of hospital stay, status of postoperative suspicion of COVID-19, and postoperative morbidity and mortality rates. All patients were evaluated for confirmed or suspected COVID-19 prior to admission to the hospital, and after discharge.

\subsection{Our approach to our adaptation to the pandemic}

At the outset of the study, all suspected COVID-19 patients presenting to the general surgery clinic were directed to the emergency department, in line with the COVID-19 Scientific Advisory Board Decision. All patients were assessed in isolation, and then moved for diagnosis and treatment to different isolated areas. The intention in this regard was to protect surgical patients presenting at the general surgery clinic from coming into contact with suspected COVID-19 cases [9]. All examinations were made wearing a surgical mask and gloves. With the rapid increase in the number of cases, every patient has started to be considered a potential carrier of COVID19, and the use of N95 or FFP (filtering facepiece) masks, eye protection and gowns has become common practice. For the patients scheduled for elective surgery, samples were sent for a COVID-19 polymerase chain reaction (PCR) test prior to hospitalization, and those with a negative test result were hospitalized. Thoracic tomography scans intended for staging were also assessed to identify viral pneumonia. Patients were admitted to surgical wards and followed-up in individual rooms, and wore masks while being taken to the operating room, with little time spent in the preoperative room. In order to reduce patient contact to a minimum prior to anaesthesia, the anaesthesia care team comprised only an anaesthesiologist and an anaesthetic technician. All patients were treated as COVID-19 positive, and the anaesthetic procedure was initiated after donning a surgical mask over an N95 mask, a surgical gown and eye protection. Intubation was made by a specialist physician using a video laryngoscope with no mask ventilation and with rapid induction, and complete nasal oxygenation was achieved. When awakening from anaesthesia, oxygen is delivered via a nasal cannula in order to avoid post-extubation masking. Recovery and awakening took place in the operating room when possible, and the patient was transferred directly to the ward, bypassing the postoperative room. Patients requiring intensive care were transferred to intensive care negative pressure isolation rooms, accompanied by the anaesthesiologist, within a different corridor. The surgical team donned fluid-repellent gowns, surgical masks over N95, eye protection and gloves (double) as personal protective equipment (PPSE). Postoperative visits to the patient rooms were limited to one physician and one nurse, keeping the number of visiting personnel to a minimum, with N95 masks and surgical gowns donned and hand hygiene administered upon entry to and departure from each room. A 14-day selfisolation period was recommended for discharged patients, who were advised to apply to the hospital again in the event of fever, cough or shortness of breath.

\subsection{Statistical analysis}

The descriptive statistics garnered for the study included mean, standard deviation, median, minimum, maximum, frequency and ratio. The SPSS 22.0 program was used for the data analyses.

\section{Results}

A total of 30 elective cancer patients underwent operations between the specified dates, of which four with breast cancer, one with thyroid cancer, two with gastric cancer, one with pancreatic cancer, three undergoing emergency operations due to ileus, and one who underwent a diagnostic laparotomy without resection were excluded from the study. A total of 18 patients underwent resection in accordance with oncological principles with a diagnosis of colorectal malignancy. Table 1 details the comorbidities, preoperative laboratory values, preoperative imaging, type of anaesthesia, duration of operation, length of hospital stay, postoperative complications and mortality of the patients. Of the total, 11 patients had comorbidities, and six had multiple comorbidities. None of the operated patients had a preoperative suspicion of COVID-19 nor a contact history. Of the total study sample, 15 patients underwent preoperative polymerase chain reaction (PCR) tests, the results of which were all negative. One patient with postoperative fever and shortness of breath was assessed with a thoracic computed tomography (CT) and COVID-19 PCR tests, however the thoracic CT showed no pulmonary involvement of COVID19, and the COVID-19 PCR test result was negative. The patient was discharged after the fever and cough regressed. Table 2 details the surgeries performed on the patients and the associated indications. No suspicions or signs of COVID19 emerged during the 14-day follow-up of the discharged patients, and none developed signs or symptoms of COVID19 within the 14 days following discharge.

\section{Discussion}

The present study identifies the changes adopted in the approaches to oncological surgery in the presence of COVID-19, which is spreading rapidly around the world and within our 
TA B L E 1. The general characteristics and demographic data.

\begin{tabular}{|c|c|c|c|c|c|}
\hline & Range (min-max) & Median & \multicolumn{3}{|c|}{ Mean $\pm \mathbf{S D} / \mathbf{n}-\%$} \\
\hline Age & $46-75$ & 62 & 59,8 & \pm & 8.8 \\
\hline Over 65 years old & & & 6 & & $33 \%$ \\
\hline Male & & & 8 & & $44.40 \%$ \\
\hline Female & & & 10 & & $55.60 \%$ \\
\hline Operation time (minutes) & $82-390$ & 235 & 228.5 & \pm & 87.5 \\
\hline Duration of stay (days) & $6-23$ & 8.5 & 10.4 & \pm & 5.2 \\
\hline Comorbidity & & & 11 & & $61.10 \%$ \\
\hline Preoperative malignant pathology & & & 18 & & $100 \%$ \\
\hline Preoperative neoadjuvant therapy & & & 4 & & $22.20 \%$ \\
\hline Preoperative chest $x$-ray & & & 18 & & $100 \%$ \\
\hline Preoperative torax tomography & & & 18 & & $100 \%$ \\
\hline Suspected preoperative CT COVID-19 & & & 0 & & $0 \%$ \\
\hline Preoperative COVID-19 test & & & 15 & & $83.30 \%$ \\
\hline COVID-19 test + & & & 0 & & $0 \%$ \\
\hline Anaesthesia type (general) & & & 18 & & $100 \%$ \\
\hline Type of surgery & & & & & \\
\hline Laparoscopic & & & 3 & & $16.70 \%$ \\
\hline Open & & & 15 & & $83.30 \%$ \\
\hline Need for postoperative intensive care & & & 5 & & $27.80 \%$ \\
\hline Postoperative intubation & & & 0 & & $0 \%$ \\
\hline Wound infection & & & 4 & & $22.20 \%$ \\
\hline Suspected postoperative COVID-19 & & & 1 & & $5.60 \%$ \\
\hline Postoperative COVID-19 test & & & 1 & & $5.60 \%$ \\
\hline Preoperative laboratory parameters & & & & & \\
\hline Preoperative WBC & $4.1-11.4$ & 7.5 & 7.6 & \pm & 1.8 \\
\hline Preoperative lymphocyte & $0.6-4$ & 2 & 2 & \pm & 0.9 \\
\hline Preoperative neutrophil & $2.6-9.3$ & 4.2 & 4.7 & \pm & 1.9 \\
\hline Preoperative CRP & $1.1-10.9$ & 2.7 & 3.5 & \pm & 2.6 \\
\hline Preoperative albumin & $2.6-4.4$ & 3.6 & 3.5 & \pm & 0.5 \\
\hline
\end{tabular}

Min-max = minimum-maximum; $C T=$ computed tomography; $W B C=$ white blood cell;

$C R P=$ c-reactive protein $; S D=$ standard deviation.

country, and is affecting the entire healthcare system. The 310 -year survival of colorectal cancer patients is lower when treatment is initiated 90 days after diagnosis than in those who begin treatment in due time, and similar findings have been reported for other cancer types [10-12]. Several countries and regions have imposed a reduction on cancer diagnoses and surgical procedures in order to ease the allocation of further resources to COVID-19 patients, and to avoid the risk of infection [13]. It now seems very unlikely that the pandemic will be resolved within weeks, leading to various opinions and plans to emerge in clinical practice [14]. Curative surgery is said to be the most important and effective treatment for many gastrointestinal cancers, and there have been recommendations that such surgeries be carried out regardless of the pandemic after ensuring the necessary precautions are taken [15]. Preoperative chemotherapy is recommended prior to surgery in locally advanced and advanced cancers due to the benefit of neoadjuvant therapy [16]. Since our hospital is a tertiary training and research hospital, cancer operations are performed following a routine multidisciplinary approach. Rectal cancer patients underwent surgery after undergoing neoadjuvant therapy, the benefits of which are well known in this patient group. Since surgery is considered the optimum approach in cases of colon cancer, non-metastatic patients underwent direct surgical treatments. Delays in the treatment of colorectal malignancies, as well as advanced stage cancers or ileus, can be life-threatening. Following the declaration of the pandemic, the issue of colorectal malignancies was discussed by the Oncological Council, and patients indicated for surgery were operated on without delay after the necessary measures were taken. The absence of suspicion of COVID-19 in postoperative patients suggests that such surgeries should 
TA B LE 2. Patient diagnoses and types of surgery.

\begin{tabular}{lcc}
\hline Operation indication & Operation procedures & Patient $\mathbf{~ n}^{\mathbf{0}}$ \\
\hline Malignancy rectum & Low anterior resection + ileostomy & 5 \\
\hline Malignancy sigmoid colon & Anterior resection & 7 \\
\hline Malignancy transverse colon & Extended right hemicolectomy & 2 \\
\hline Malignancy right colon & Right hemicolectomy & 2 \\
\hline Gynecological malignancy colon invasion & Total colectomy & 1 \\
RCC sigmoid colon invasion & Anterior resection & 1 \\
\hline Total & & $\mathbf{1 8}$ \\
\hline
\end{tabular}

$R C C=$ renal cell carcinoma .

not be postponed unnecessarily.

As is the case with all elective procedures, the number of gastrointestinal endoscopy and colonoscopy procedures being carried has witnessed a significant reduction following the announcement of the pandemic [17]. Similarly, in a survey carried out in North American, $65 \%$ of centers were found to be carrying out less than $10 \%$ of their usual volume of surgeries [18]. In our hospital, with the onset of the pandemic, only emergency patients and patients with suspected malignancies undergo colonoscopy, and only after the personal protective measures are taken. This has led to a reduction in the number of colonoscopies carried out, similarly to other centers.

Data on the results of elective colorectal surgeries and COVID 19 transmission risk rates in facilities assigned as pandemic hospitals is still limited in literature. Sorrentino et al [19]. identified no significant difference in contagiousness following colorectal surgery among patients from a pandemic region and those not from a pandemic region.

Laparoscopy is a common approach in colorectal cancers, being less traumatic and associated with better postoperative recovery [20]. That said, whether surgical procedures should be performed using laparoscopic or conventional methods during the COVID-19 pandemic is still a matter of discussion. As is known, the available experience is the early results from case reports or case series [1]. In a study by Yu et al [8], SARS-CoV2 was reported to be transmitted through droplets and contact, although fecal-oral and aerosol transmission could not be ruled out. It can thus be concluded that laparoscopic surgeries can be performed on colon cancer patients infected with COVID-19, as long as the laparoscopic gases are well managed. Likewise, Morris et al [21]. determined that the laparoscopic approach could be used in gynecological cases, as the risk of COVID19 in such surgeries has yet to be ascertained. In the present study, three cases underwent laparoscopy at the beginning of the process, but with the rapid spread of COVID-19, open surgery started to be preferred, considering that all patients could be asymptomatic, even when tomography scans were negative. Since the laparoscopic approach takes longer than open surgery, open surgeries were preferred later as a means of minimizing the risk of infection.

A review by Di Saverio et al [22] suggested that all patients scheduled for surgery should undergo testing for COVID-19. In the present study, no preoperative PCR was requested for three patients, given the lack of any published guidelines or recommendations in the early days of the outbreak. Tests were later requested in accordance with published recommendations, and patients started to be admitted to the surgical ward based on their test results [1,3]. In the earliest days of the outbreak, no PCR tests were available at our facility, despite being designated a pandemic hospital, and this resulted in the first three patients in the study undergoing surgery without PCR testing. Some asymptomatic COVID-19 patients may be identified through tomography findings prior to the results of PCR tests being obtained [23]. The use of preoperative thoracic tomography for staging in all malignant patients provides important data on early asymptomatic patients. No viral pneumonia was identified in any of the preoperative thoracic tomography scans of the cases in the present study. In one patient with postoperative suspicion of COVID-19, the PCR test and thoracic tomography were repeated, but the patient was discharged without event following the return of negative results and an improved clinical presentation.

The evaluation only of patients with colorectal cancer may be construed as a limitation of the present study, however, since patients with colorectal cancer are candidates for mechanical intestinal obstruction in the early stage, and malignancies such as breast or pancreas are fortunately not left untreated with modalities such as neoadjuvant chemoradiotherapy, the surgical treatment of our patients with non-colorectal cancers could be postponed during the period of the study.

\section{Conclusions}

As reported in the present study, the rapid spread of the COVID-19 pandemic made it necessary to carry out COVID19 treatments and cancer surgery alongside each other, with no standardized algorithms or comprehensive guidelines, in the early days of the pandemic. We believe that risk can be minimized through the use of personal protective equipment (PPE), by conducting pre-hospitalization PCR tests for COVID-19 with negative results, the assurance of minimum contact between healthcare personnel and the patient, and the isolation of surgical oncology wards from pandemic wards. Our assessment of the cases in the present study found that patients undergoing colorectal surgery and those with COVID-19 infections can be treated successfully, without affecting each other, if proper isolation approaches are followed, supporting the management of the two patient groups in the same hospital. These successful outcomes were supported by a 14-day follow-up after discharge. 


\section{ACKNOWLEDGEMENTS}

Thanks to all the peer reviewers and editors for their opinions and suggestions.

\section{CONFLICTS OF INTEREST}

The author has no conflicts of interest to declare.

\section{ETHICAL APPROVAL}

Approval for this study was obtained from the ethics commission of the University of Health Sciences of the Haseki Research and Education hospital, with approval $\mathrm{n}^{\mathrm{o}}$ : 89-2020, dated 03.06.2020.

\section{REFERENCES}

[1] Kamer E, Çolak T. What to do when a patient infected with COVID-19 needs an operation: a pre-surgery, peri-surgery and post-surgery guide. Turk J Colorectal Dis. 2020;30:1-8.

[2] Dost B, Koksal E, Terzi Ö, et al. Attitudes of anaesthesiology specialists and residents toward patients infected with the novel coronavirus (COVID-19): a national survey study. Surg Infect (Larchmt). 2020;21:350-356

[3] Karaca AS, Özmen MM, Uçar AD, et al. General surgery operating room practice in patients with COVID-19. Turk J Surg. 2020;36:I-V.

[4] Ti LK, Ang LS, Foong TW, et al. What we do when a COVID-19 patient needs an operation: operating room preparation and guidance. Can J Anesth. 2020;67:756-758.

[5] Turkiye Cumhuriyeti Sağlık Bakanlığı. Türkiye Coronavirus enfeksiyonu verileri. Availablefrom: https://covid19.saglik.gov.tr/. Accessed in 2020 (Jun 26).

[6] Gok AFK, Eryılmaz M, Ozmen MM, et al. COVID-19 Pandemisi Sırasında Travma ve Acil Cerrahi Uygulamaları İçin Öneriler [Recommendations for trauma and emergency general surgery practice during COVID-19 pandemic]. Ulus Travma Acil Cerrahi Derg. 2020;26:335342 .

[7] Bozkurt H, Gür HÜ, Akıncı M, et al. Evaluation of patients undergoing emergency surgery in a COVID-19 pandemic hospital: a crosssectional study. Sao Paulo Med. J. Published online ahead of print. doi: 10.1590/1516-3180.2020.0181.R1.13052020.

[8] Yu GY, Lou Z, Zhang W. Several suggestion of operation for colorectal cancer under the outbreak of coronavirus disease 19 in China. Zhonghua Wei Chang Wai Ke Za Zhi. 2020;23:9-11.

[9] Turkiye Cumhuriyeti Sağlık Bakanlığı Pandemi Hastaneleri. Available from: https : / /hasta.saglik.gov .tr/. Accessed in 2020 (Jun 26).

[10] Roder D, Karapetis CS, Olver I, et al. Time from diagnosis to treatment of colorectal cancer in a South Australian clinical registry cohort: how it varies and relates to survival. BMJ Open. 2019;9:e031421.
[11] Aseem R, Warren O, Mills S, et al. Adjusting to the COVID-19 pandemic: challenges and opportunities of frontline colorectal cancer teams in the UK. Int J Colorectal Dis. 2020;35:1783-1785.

[12] Pellino G, Spinelli A. How Coronavirus Disease 2019 Outbreak Is Impacting Colorectal Cancer Patients in Italy: A Long Shadow Beyond Infection. Dis Colon Rectum. 2020;63:720-722.

[13] Aminian A, Safari S, Razeghian-Jahromi A, et al. COVID-19 outbreak and surgical practice: unexpected fatality in perioperative period. Ann Surg. 2020;272:e27-e29.

[14] Ghignone F, Mohan HM, Montroni I. Cancer surgery in a time of COVID19: Many questions, few certainties. Eur J Surg Oncol. 2020;46:11961197.

[15] American College of Surgeons. COVID 19: elective case triage guidelines for surgical care. 2020. Available from: https : / www . facs . org/covid-19/clinical-guidance/elective-case. Accessed in 2020 (Jun 26).

[16] Habr-Gama A, Perez RO, Sabbaga J, et al. Increasing the rates of complete response to neoadjuvant chemoradiotherapy for distal rectal cancer: results of a prospective study using additional chemotherapy during the resting period. Dis Colon Rectum. 2009;52:1927-34.

[17] Lui T, Leung K, Guo CG, et al. Impacts of COVID-19 Pandemic on Gastrointestinal Endoscopy Volume and Diagnosis of Gastric and Colorectal Cancers: A Population-based Study. Gastroenterology. doi: https://doi.org/10.1053/ j.gastro.2020.05.037.

[18] Forbes N, et al. Gastroenterology 2020 May 3. pii:S0016-5085(20)305928. doi: 10.1053/j.gastro.2020.04.071.

[19] Sorrentino L, Guaglio M, Cosimelli M. Elective colorectal cancer surgery at the oncologic hub of Lombardy inside a pandemic COVID-19 area. J Surg Oncol. 2020;122:117-119.

[20] Pence HH, Sert ÖZ, Bozkurt H, et al. The Effects of Laparoscopic Colon Surgery on Postoperative Renal Functions. Med J Bakirkoy. 2019;15:310315 .

[21] Morris SN, Fader AN, Milad MP, Dionisi HJ. Understanding the "scope" of the problem: why laparoscopy is considered safe during the COVID-19 pandemic. J Minim Invasive Gynecol. 2020;27:789-91.

[22] Di Saverio S, Sibilio A, Giorgini E, et al. The NOTA Study (Non Operative Treatment for Acute Appendicitis): prospective study on the efficacy and safety of antibiotics (amoxicillin and clavulanic acid) for treating patients with right lower quadrant abdominal pain and long-term follow-up of conservatively treated suspected appendicitis. Ann Surg. 2014;260:109-17.

[23] $\mathrm{Hu} \mathrm{Z}$, Song $\mathrm{C}, \mathrm{Xu} \mathrm{C}$, et al. Clinical Characteristics of 24 Asymptomatic Infections With COVID-19 Screened Among Close Contacts in Nanjing, China. Sci China Life Sci. 2020;63:706-11.

How to cite this article: Muzaffer Akinci, Hilmi Bozkurt, Hüda Ümit Gür, Adil Koyuncu, Mahmut Said Değerli, Sercan Yüksel, Orçun Alpay, Ahmet Kocakuşak. Evaluation of Colorectal Surgical Patients and Treatment Modalities in a COVID-19 Pandemic Hospital: A Cross-sectional Study. Signa Vitae. 2020;16(2):155-159. doi:10.22514/sv.2020.16.0059. 